\title{
Research of new structure super fast recovery power diode*
}

\author{
Li Ma ${ }^{1, a}$, Linnan Chen ${ }^{2, b}$,Yong Gao ${ }^{3, c}$ \\ ${ }^{1,2}$ School of Science, Xi'an University of Technology, Shaanxi 710054, China; \\ ${ }^{3}$ Xi'an University of Technology, Shaanxi 710048, China. \\ amali@xaut.edu.cn, bchenlinnan903@sina.com, cgaoy@xaut.edu.cn
}

Keywords: fast recovery diode, reverse recovery, device modeling.

\begin{abstract}
This study aims to greatly improve the reverse recovery characteristics of the fast recovery diode by changing the diode structure. Through adjusting the doping concentration, thickness and width of anode $\mathrm{p}$ area and considering its impact on device characteristics, it effectively reduces the reverse recovery current peak and reverse recovery time. Therefore, it can better meet requirements of soft quick recovery, high breakdown voltage performance of the power diode which are applied in high frequency, power electronic circuits.
\end{abstract}

\section{Introduction}

Power electronic device which can be applied to achieve energy efficient and precise control is one of power semiconductor devices, it is the foundation of the power electronic technology ${ }^{[1-2]}$. With the continuous development of power electronic technology, the development of power device is toward the direction of high pressure, high power, high frequency, integration and intelligent. Therefore, fast recovery diode should have high compression $\left(V_{B}\right)$, low positive voltage drop $\left(V_{F}\right)$, small reverse recovery current $\left(\mathrm{I}_{\mathrm{RR}}\right)$, short reverse recovery time $\left(\mathrm{t}_{\mathrm{RR}}\right)$, big softness factor $(\mathrm{S})$, etc ${ }^{[3]}$.

Don't shorten extra carrier lifetime. Reverse recovery characteristics of power p-i-n diode also can be improved obviously through pn junction structure changes. So we have derived the SSD (Static Screened Diode). The significant difference between this structure and conventional p-i-n diode is its p layer that does not have uniform thickness and impurity concentration. Instead of putting the uniform distribution of high concentration and deep $\mathrm{p}^{+}$micro area embed in the lower concentration of shallow junction p thin layer. Because of extra hole injection ratio of p-i-n junction associated with doping concentration of $\mathrm{p}$ layer, thus the SSD is equivalent to two micro p-i-n with different injection ratio in parallel. In this way, drift region's high concentration of holes injected by the high injection ratio of $\mathrm{p}^{+}$-n junction spread around will like MPS (Merged p-i-n Schottky) injection holes, and weak extra carrier storage effect. The reverse recovery characteristics have remarkable improvement on account of the storage charge density decreased. The compromise effect can be compared with the MPS in the forward voltage drop and reverse recovery current ${ }^{[4]}$.

\section{Device structure}

Design of the power device could withstand high voltage in the depletion layer on the interface of the PN junction, metal-semiconductor (schottky barrier) contact or metal-oxide-semiconductor $(\mathrm{MOS})^{[5]}$. Low doping region under the PN junction (shallow junction depth and high surface doping concentration) have a opposite conductive type. Depletion region mainly expand to low doping region, it can be used as abrupt junction. And punch-through p-i-n diode after reducing the thickness of the drift region, is not only beneficial to reduce the on-state voltage drop, but also beneficial to reduce the reverse recovery power consumption resulting by storage charge and the storage charge ${ }^{[6]}$. Because punch-through design can use thinner low doping concentration depletion region to

\footnotetext{
* 1. The National Natural Science Foundation of China (Grant no. 51177133)

2.The Special Scientific Research Program of the Education Bureau of Shaanxi Province, China (Grant no. $2013 J K 11$ 05).
} 
withstand voltage, so this article analyzed structure and technological parameters by means of simulation on the basis of punch-through diode.

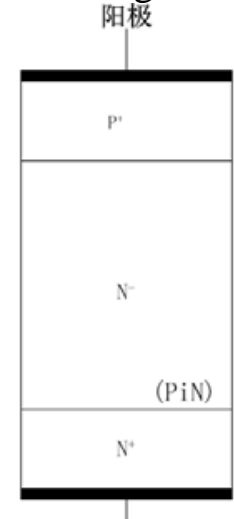

阴极

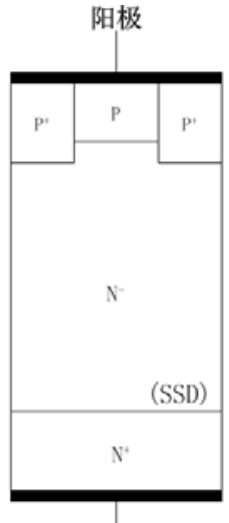

阴极

Fig. 1 The PiN and SSD device structures

This paper presents a new structure that adding $\mathrm{p}$ area to the anode $\mathrm{p}^{+}$area, without altering the diode reverse blocking characteristics, improved the diode reverse recovery characteristics effectively and reduced the reverse recovery time. As shown in fig. 1, a new structure of the diode is the $\mathrm{P}^{+}$area of the traditional $\mathrm{PiN}$ diode that have changed to $\mathrm{P}^{+} / \mathrm{P} / \mathrm{P}^{+}$area, including $\mathrm{P}^{+}$and $\mathrm{P}$ form the discretion of the lateral $\mathrm{P}^{+}-\mathrm{P}$ junction. The depletion region's thickness of the new structure diode is 10um, thickness of cathode area $\mathrm{N}^{+}$is $4 \mathrm{um}$, thickness of anode $\mathrm{P}^{+}$area is 4um, thickness and width of anode $\mathrm{P}$ area can be adjusted, discussed in the following analysis.

Simulation of the SSD diode compared with conventional PiN diode with same size and parameter can be concluded that the result is shown in fig. 2. This new structure of SSD diode can obtain a faster switching speed, more soft recovery feature and low forward voltage drop. It significantly improves the comprehensive properties of the device.

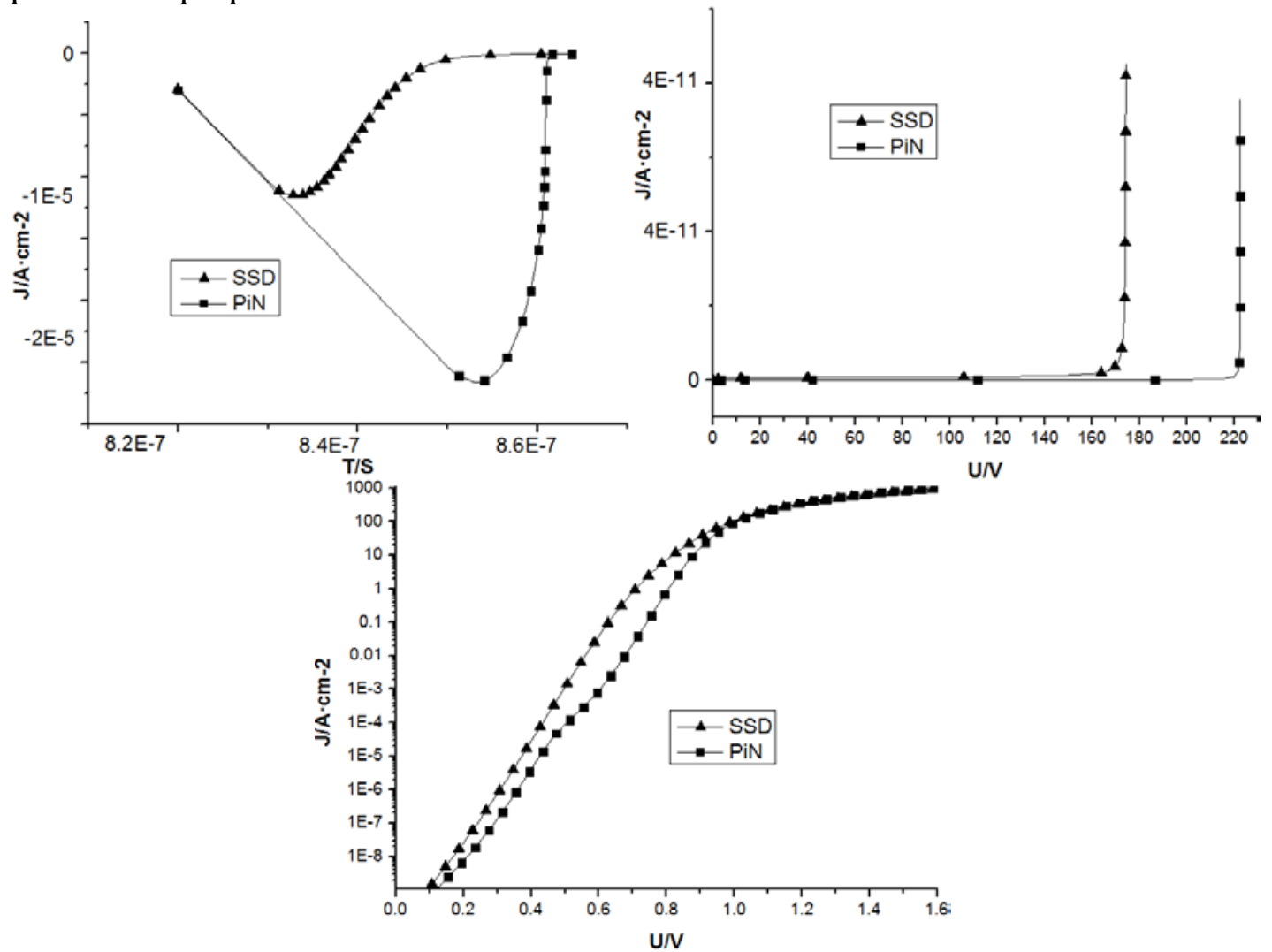

Fig. 2 Comparison of conventional pin diode with SSD diode 


\section{Simulation analysis and optimization}

We have adopted electronic device simulation software ISE-TCAD. Using MDRAW and DESSIS tools of software carry on simulation test in structure dynamic and static properties of SSD power diode. Optimizing the process parameters and structure parameters extracts the optimal parameters to determine the final process.

Basic device, unless otherwise noted, width is $12 \mathrm{um}$, thickness is $18 \mathrm{um}$. The thickness of the $\mathrm{P}^{+}$ region is $4 \mathrm{um}$, doping concentration is $10^{19} / \mathrm{cm}^{3}$. The thickness of drift region $\mathrm{N}^{-}$is $10 \mathrm{um}$, doping concentration is $10^{15} / \mathrm{cm}^{3}$. The thickness of the $\mathrm{n}^{+}$region is 4um, doping concentration is $10^{20} / \mathrm{cm}^{3}$.

The influence of anode $p$ area concentration on the diode characteristic. As shown in fig. 3 , the influence of $\mathrm{P}$ area doping concentration to diode characteristics, it can be seen that the reverse recovery time and the reverse recovery current peak decrease as the $\mathrm{P}$ area doping concentration reduce gradually. Because drift region filled with excess carrier as well as hindered the establishment of space charge at the beginning of the turn-off process. So the voltage across the diode remains a small value when the reverse begins. Then the current gradually reduced to zero until reversed, and the reverse current in size and down approach by influence of carrier diffusion and the composite effect of voltage-sustaining layer. Obviously, the greater doping concentration of $\mathrm{p}$ area is not conducive to the electron recombination or electron diffusion, also makes the foreign reverse current shows greater peak.

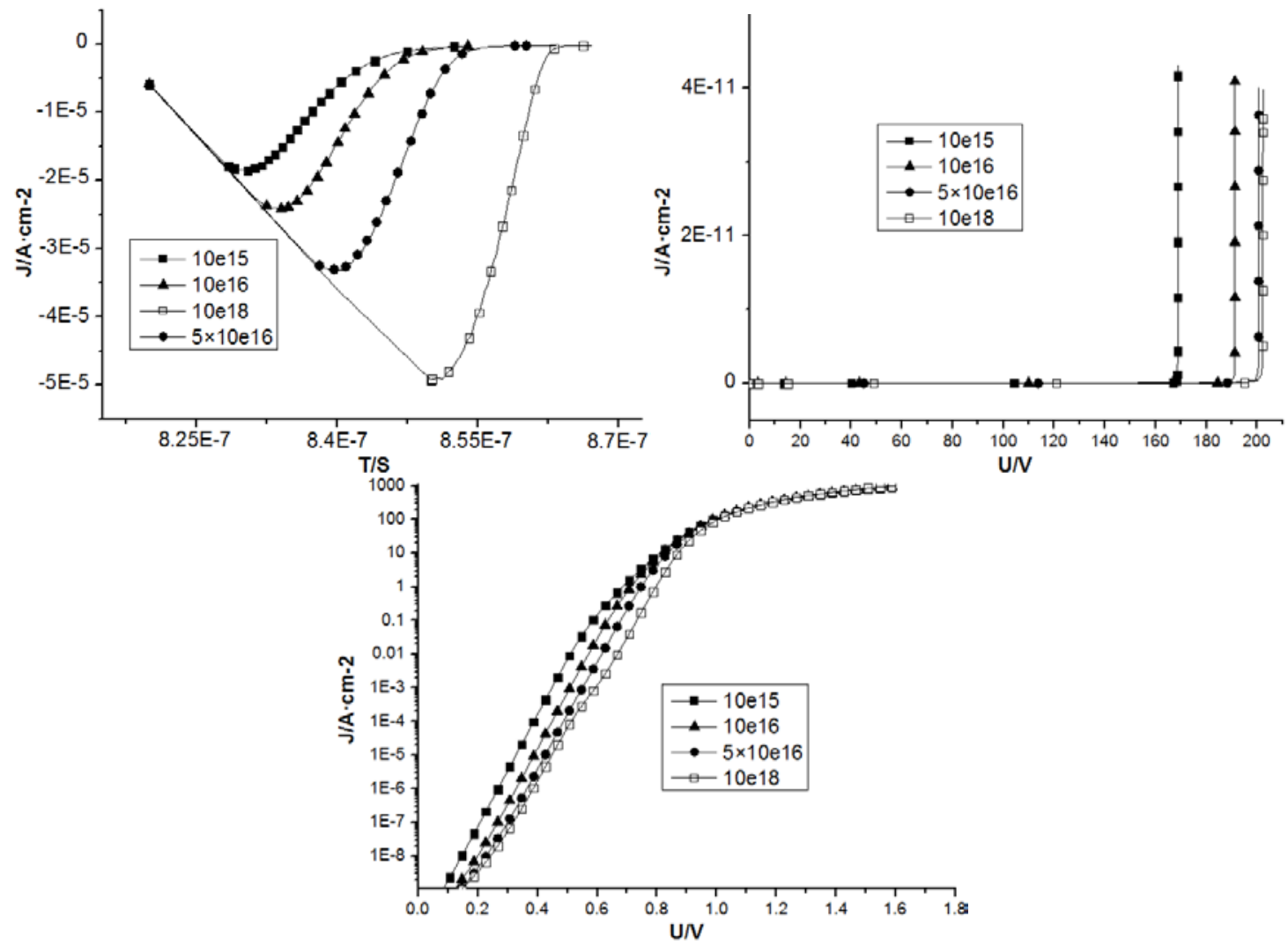

Fig. 3 The influence of $\mathrm{P}$ area doping concentration to diode characteristics

When the concentration of $\mathrm{p}$ area is less than $5 \times 10^{16} / \mathrm{cm}^{3}$, the breakdown voltage will change a lot. On the contrary, if the concentration of $\mathrm{p}$ area is greater than it, the breakdown voltage change is very small. It can be seen from the above, the positive characteristics of diode under the condition of the small current density, positive pressure drop increased slightly with the increase of the doping concentration. To sum up, taking the consideration of the difficult tradeoffs, the research chooses $10^{16} / \mathrm{cm}^{3}$ doping concentration of $\mathrm{p}$ area.

The influence of anode $\mathbf{p}$ area width on the diode characteristic. As shown in figure 4 , the influence of $\mathrm{P}$ area width to diode characteristics, it can be seen the reverse recovery time and reverse recovery current peak decrease as anode $\mathrm{P}$ area width increase gradually. Since the reverse current's size and down approach are influenced by the carrier diffusion and the composite effect in the 
voltage-sustaining layer. Apparently, the bigger width of $\mathrm{P}$ area is more advantageous to electronic composite or diffusion, reducing the reverse current peak. Reverse breakdown voltage slightly increase as the width of the $\mathrm{P}$ area decrease, it can be seen from above. Forward characteristic of device has not deterred particularly evident change for changing $\mathrm{p}$ area width. when the current density is small, the diode forward voltage drop decrease with the increase of $\mathrm{p}$ area width. In conclusion, in order to increase the reverse recovery characteristic and decrease reverse blocking voltage and forward voltage drop, increasing the $\mathrm{P}$ region width. So $\mathrm{P}$ area width is 6um, the performance of low positive voltage drop and high blocking voltage and fast recovery is best.

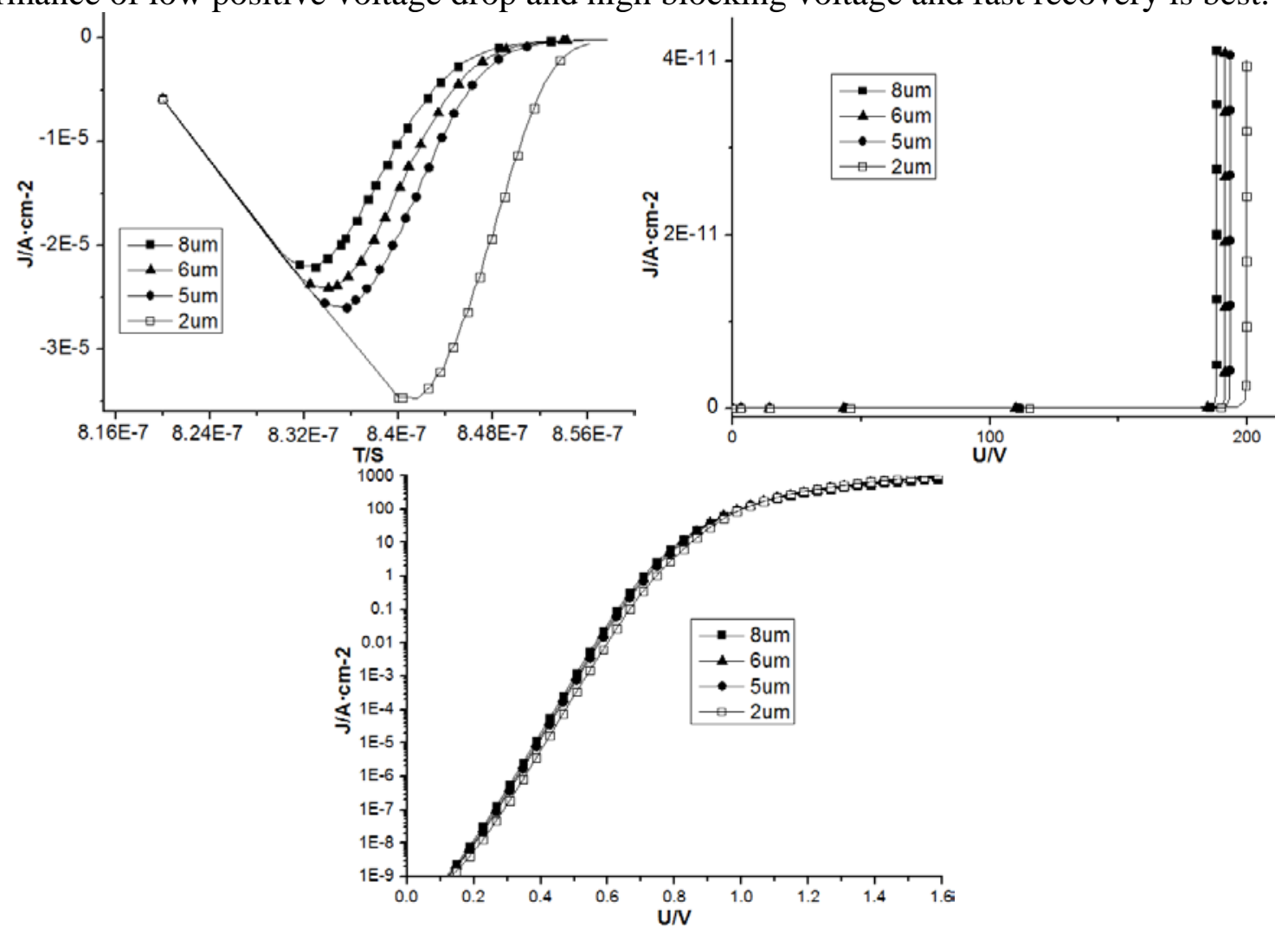

Fig. 4 The influence of $\mathrm{P}$ area width to diode characteristics

The influence of anode $\mathbf{p}$ area thickness on the diode characteristic. As shown in figure 5 , the influence of $\mathrm{P}$ area thickness to diode characteristics, it can be seen the reverse recovery time and reverse recovery current peak decrease as $\mathrm{P}$ area thickness decrease gradually. Reverse breakdown voltage decrease as the thickness of the $\mathrm{P}$ area decrease can be seen from the above. Forward characteristic of device has not deterred particularly evident change for $\mathrm{p}$ area width change. Obviously, $\mathrm{P}$ area thickness is 3um, the performance of low positive voltage drop and high blocking voltage and fast recovery is best.
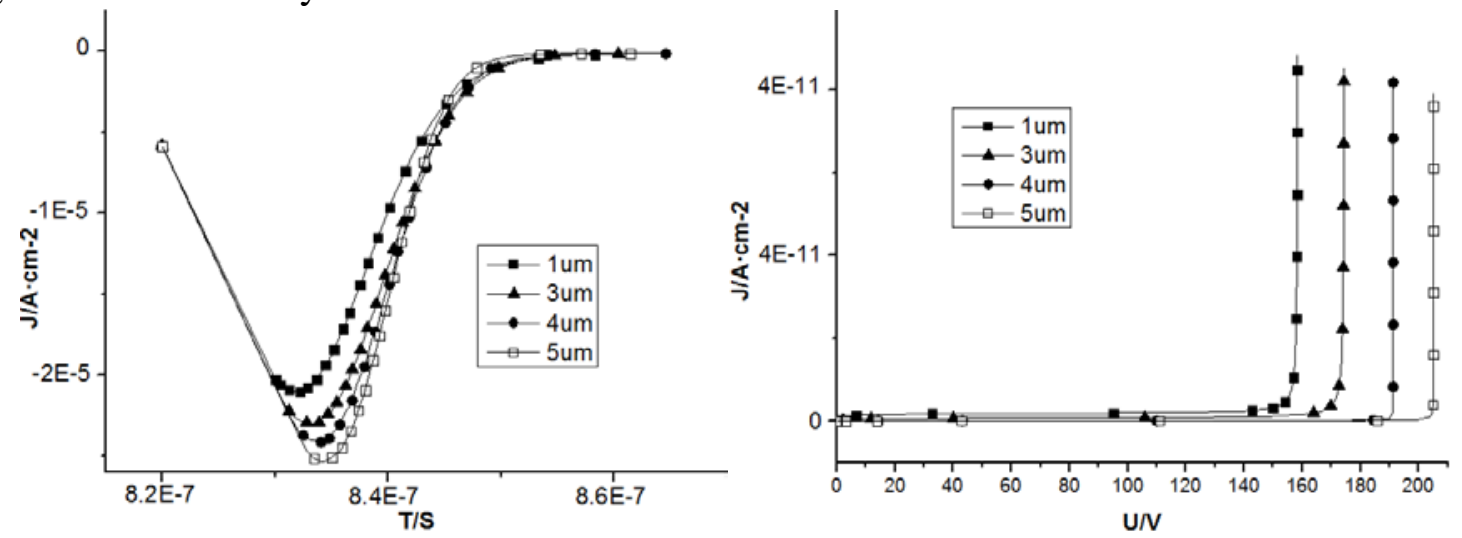


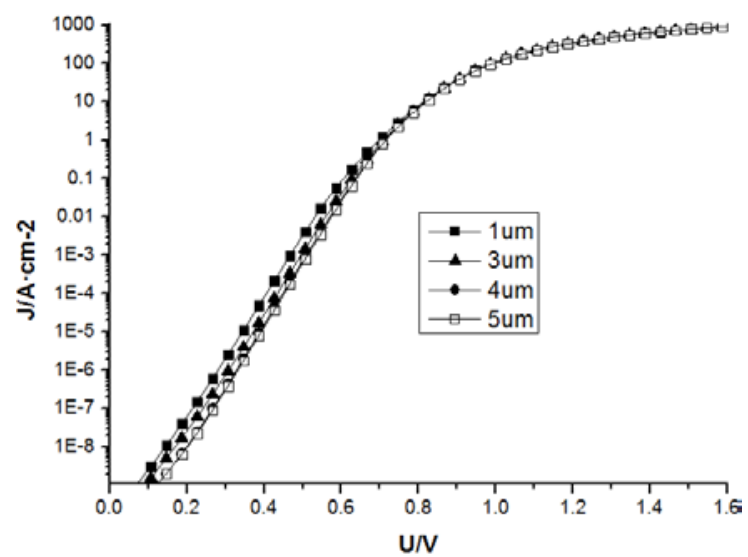

Fig. 5 The influence of $\mathrm{P}$ area thickness to diode characteristics

\section{Summary}

This paper presents concrete structure parameter of a new structure fast recovery of SSD diode, and its electrical properties than conventional PiN diode has a great improvement through simulation verification. This new structure of SSD diode can obtain a faster switching speed, more soft recovery feature and low forward voltage drop. It significantly improve the comprehensive properties of the device.

\section{References}

[1] Baliga B J. Power Semiconductor Devices [M], PWS, Boston, 1995. Reference to a book:

[2] Porst A. Ultimate limits of an IGBT (MCT) for high-voltage applications in conjunction with a diode [C]. In Proc. IEEE Int. Symp. Power Semiconductor Devices and ICs (ISPSD), 1994, 163-167.

[3] Pierric Gueguen, How power electronics will reshape to meet the 21th century challenges[C]. In Proc. IEEE Int. Symp. Power Semiconductor Devices and ICs (ISPSD), 2015, pp. 17-20.

[4] Ogata S, Miyanagi Y, Nakayama K, Tanaka A, Asano K. 5kV class 4H-SiC PiN Diode with Low Voltage Overshoot during Forward Recovery for High Frequency Inverter[C]. IEEE 23rd International Symposium on Power Semiconductor Devices and ICs (ISPSD), San Diego, CA, 2011, pp. 296-299.

[5] Fumihiko Hirose, Kazunari Kurita, Yutaka Takahashi and Masashi Mukaida, Operation Mechanism on SiGe/Si/Si PIN Diodes Explained Using Numerical Simulation. Electrochemical and Solid-State Letters [J], 2005, 8(7):160-163.

[6] B.Jayant Baliga. Fundamentals of Power Semiconductor Devices [M], Springer US, 2008: 100-104. 